\title{
Recent advances of laparoscopic approach in adnexal torsion of premenarcheal girls
}

\author{
Costas Panayotidis • Michelle Nisolle
}

Received: 1 April 2007 / Accepted: 10 May 2007 / Published online: 26 June 2007

(C) Springer-Verlag 2007

\begin{abstract}
The adnexal torsion is an uncommon gynaecological emergency; however, it must be excluded in young girls in order to conserve their future ovarian function and fertility. The diagnosis of adnexal torsion is particularly difficult in girls before the menarche during which time the clinical examination is very delicate. We review the recent literature and laparoscopic advances in this domain and propose a management algorithm.
\end{abstract}

Keywords Adnexal torsion · Detorsion · Laparoscopy · Ovarian cryopreservation $\cdot$ Premenarcheal girls

\section{Background}

Clinical features of adnexal torsion can vary from vague, intermittent abdominal pains or - more frequently - severe pains, colic-type pains or constant pain in the iliac fossa, to general malaise with vomiting, agitation and abdominal rigidity with or without rebound. These signs are not specific and can be presented at this age with other, more frequent, pathological conditions, such appendicitis. Clinical suspicion of adnexal torsion should be included in the differential diagnosis for low pelvic pain in a young girl. Good practice suggests vigilance in order to exclude pos-

C. Panayotidis $(\square)$

Obstetrics and Gynaecology, University of Manchester,

Manchester, UK

e-mail: costapan@hotmail.com

M. Nisolle

Department of Gynaecology and Obstetrics,

University of Liege, Centre Hospitalier Regional Citadelle,

Boulevard du 12me De Ligne 1,

4000 Liege, Belgium sible abuse or suspected domestic violence in young girls in case of vague, nonspecific abdominal pains. The diagnosis depends on the systematic clinical approach to the young girl and how fast she will be referred to an experienced gynaecologist. Most often, these girls have been seen first by a general practitioner or have been self-referred to the accident and emergency department. Paediatric registrars or general surgeons are then in charge. Conservative management with strong analgesia and observation can delay the diagnosis. In acute abdomen, a surgical approach is immediately necessary, and again, this should be organised with the collaboration of an experienced senior gynaecologist.

Torsion of the adnexal in premenarcheal girls is most often associated with an ovarian cyst or mass. Germ-cell tumours are frequently found-in about $60 \%$ of cases $(70$ $90 \%$ are benign, mature teratomas or dermoid cysts). Other associated findings are tubal cysts, fallopian-tube torsion and serous or mucinous cystadenomas [1,2]. Malignant tumours, especially in this young age group, are associated with torsion in less than $10 \%$ of cases [3]. Anatomical factors have been advocated to explain the torsion, such as an abnormally long tube, incomplete mesosalpinx or an overly active ovary [4]. Right adnexal torsion is more commonly reported than left, with ratios varying from 3:2 or 5:1 [1-5]. Normal ovaries can be associated with up to $20-25 \%$ of cases of torsion [6,7]. Isolated fallopian-tube torsion has been reported without any other associated abnormality $[8,9]$.

Family anatomical predisposition was recently reported as a risk factor in the case of a 7-year-old girl who twice has had ovarian torsion. Her mother had a similar episode in her youth [10]. Sometimes, with or without an ovarian cyst, intense physical activity such as ballet or gymnastics has been an associated factor in cases of adnexal torsion [11]. 
Rotation of the adnexa causes tension on the vascular supply as well as causing venous congestion, and local oedema is generated in the ovarian tissue. Arterial blood flow to the ovary is compromised to differing degrees. Ischaemia with central haemorrhage and ultimately necrosis of the ovary may result in severe cases. The degree of ovarian damage may depend on the duration and the ischaemic effect on the tissues. Long-term torsion without tight strangulation may not have a similar ischaemic effect on the ovary, as in a case of very tight torsion over a brief period of time. Clinical signs and subjective pain are not associated with or predictive of the ischaemia and its consequences. The duration of ischemia necessary to provoke irreversible damage is difficult to define. However, better chances for ovarian recovery should be expected when surgical detorsion with reperfusion is achieved with the least possible delay. The effects of twisted ischaemic adnexa managed by detorsion in rodent experimental models showed better results on ovarian viability and histology when detorsion was achieved in less than $24 \mathrm{~h}$ [12].

\section{Diagnosis}

Although there are no specific signs of ovarian torsion, severe abdominal pain is most commonly associated with it. Intermittent abdominal pain can suggest torsion of the adnexa with spontaneous resolution rather subacute or acute appendicitis. More often, surgical investigation may be attempted when pain is located in the right iliac fossa for possible treatment of suspected appendicitis.

Abdominal (or transvaginal in exceptional cases of sexually active young girls) ultrasound scan (US) scan significantly helps the diagnostic orientation, especially when an ipsilateral ovarian cyst or tumour is located. The availability of US cover is paramount in accident-emergency departments. For example, in the UK, gynaecological US cover during the night or weekend may not always be possible, which is in contrast to other countries in Europe-for example, in Belgium or France where most gynaecologists and trainees in the speciality are competent in US scanning. Conservative measures with symptomatic treatment using strong analgesia and observation whilst awaiting a US scan during normal working hours delays the diagnosis, with possible deleterious consequences for the ovarian tissue. The US scan can detect solid adnexal cysts, large fluid cysts, ovarian enlargement or fluid in the pouch of Douglas [7]. Dermoid cysts can have various ultrasonographic appearances (fat, hair teeth, etc.); other differentials, such as haemorrhagic cyst or appendiceal abscess, can have a similar appearance. Ovarian oedema and transudate may provoke enlargement of the affected ovary, with multiple follicles in the cortex, in contrast to the small, contralateral ovary.
The existence of enlarged, multiple, uniform follicles in the cortex can be a significant sign, frequently associated with adnexal torsion in up to $74 \%$ of cases $[9,13,14]$. Sometimes, the US scan cannot detect any specific structure, and the ovaries have a normal aspect. Colour Doppler study can give some information about flow vascularity in the presumed area of torsion. Variable degrees of adnexa rotation or strangulation may not always modify the flow aspect in Doppler study. Normal Doppler findings cannot exclude early stage torsion [14]. More significant findings towards the suspicion of adnexal torsion are the absence of vascularisation, as well as severe hypoperfusion of either venous or arterial flow [15].

A computed tomography (CT) scan can detect an ovarian mass, multiple ovarian cystic lesions in the cortex and enlargement of the affected ovary. CT scan may be used in patients with suspected appendicitis. Sometimes, the anatomic relation of the suspected adnexal torsion with nearby structures is not clear. CT scan is not recommended when a gynaecological lesion is suspected, as a magnetic resonance imaging (MRI) scan can help the diagnosis in these cases, providing further details. MRI can detect pelvic masses and fluid in the pouch of Douglas, describe possible intracystic haemorrhage infarction and describe the content of an ovarian mass (dermoid, solid or mixed cyst) [16]. Features of adnexal torsion can be associated with tube thickening or smooth ovarian-wall thickening. Enlargement of the affected adnexa can be an early sign of twisted adnexa $[13,16]$. Associated features have been described as ipsilateral deviation of the uterus (traction effect from the adnexa) and hyperintense, small cystic lesions on T2-weighted images at the periphery cortex of the ovary adnexa. These cystic lesions can be a specific sign of torsion in $74 \%$ of twisted ovaries (oedema transudation of fluid in the peripheral follicles) $[13,16]$. Low, inhomogeneous overall signal intensity on T1-weighted and T2-weighted images with lack of gadolinium uptake is suggestive of infarction. Sometimes, beaked protrusion of the twisted mass on the side facing the uterus and continuing in a band-like structure connecting it with the uterus has been described [14]. Inflammatory syndrome with raised $\mathrm{C}$-reactive protein (CRP) and lactate dehydrogenase (LDH) is frequently seen although not specifically associated with ovarian adnexal torsion and ischaemia. Raised biological parameters of inflammation can raise suspicions leading to the diagnosis of ovarian torsion.

\section{Patient approach, counselling difficulties and consent issues}

Clinical examination is delicate in a premenarcheal girl. Clinical assessment is limited and is based on abdominal 
examination with inspection and palpation, blood pressure monitoring and temperature measurement. No vaginal examination usually is possible because the patient at this age is usually virgo. Taking the history from a young girl, who may be afraid of the "hostile, unfamiliar environment of the hospital", is not reliable or precise. Parents, and particularly the mother, may help to give information regarding the history and clinical signs of the daughter. However, it is not uncommon that the parents' own fears and concerns will cause stress and pressure for the examining physician.

Examination of a young girl, especially when she is in pain, should be undertaken carefully by an experienced gynaecologist. Appropriate contact and a trustful relationship should be obtained. Clear information about management consent issues in case of operation are a point that should be emphasised, following the guidelines of the Royal College of Obstetricians and Gynaecologists and the General Medical Council. Counselling, patient confidentiality and a parent's responsibility for consent are delicate issues. In an emergency, counselling skills are necessary in order to explain clearly pain management plan and each possible outcome. Figure 1 provides a management algorithm for adnexal torsion in premenarcheal girls.

Delay and latent time between initial symptoms and medical advice is something that needs to be taken into consideration. It is not only the time in the hospital that counts toward eventual serious adnexal ischaemia.

\section{Discussion}

Management

It is essential to exclude pregnancy or more frequent pathology of abdominal pain. Conservative management can be suggested when an adnexal mass is excluded or there is no sign of malignancy (imaging techniques). During the time of imaging examination by US scan or MRI, a trial of conservative management with strong analgesia is indicated in order to provide comfort to the patient. If pain resolves in
Fig. 1 Algorithm for an operational approach to adnexal torsion in premenarcheal girls. * Ovariopexy: unilateral or bilateral to be consider in the first laparoscopy or in a second-look checkup. ** Ovarian biopsy, possible ovarian cryopreservation in the future (?).

*** Adnexectomy in case of nonrecovering gangrenous ovary or complication during operation

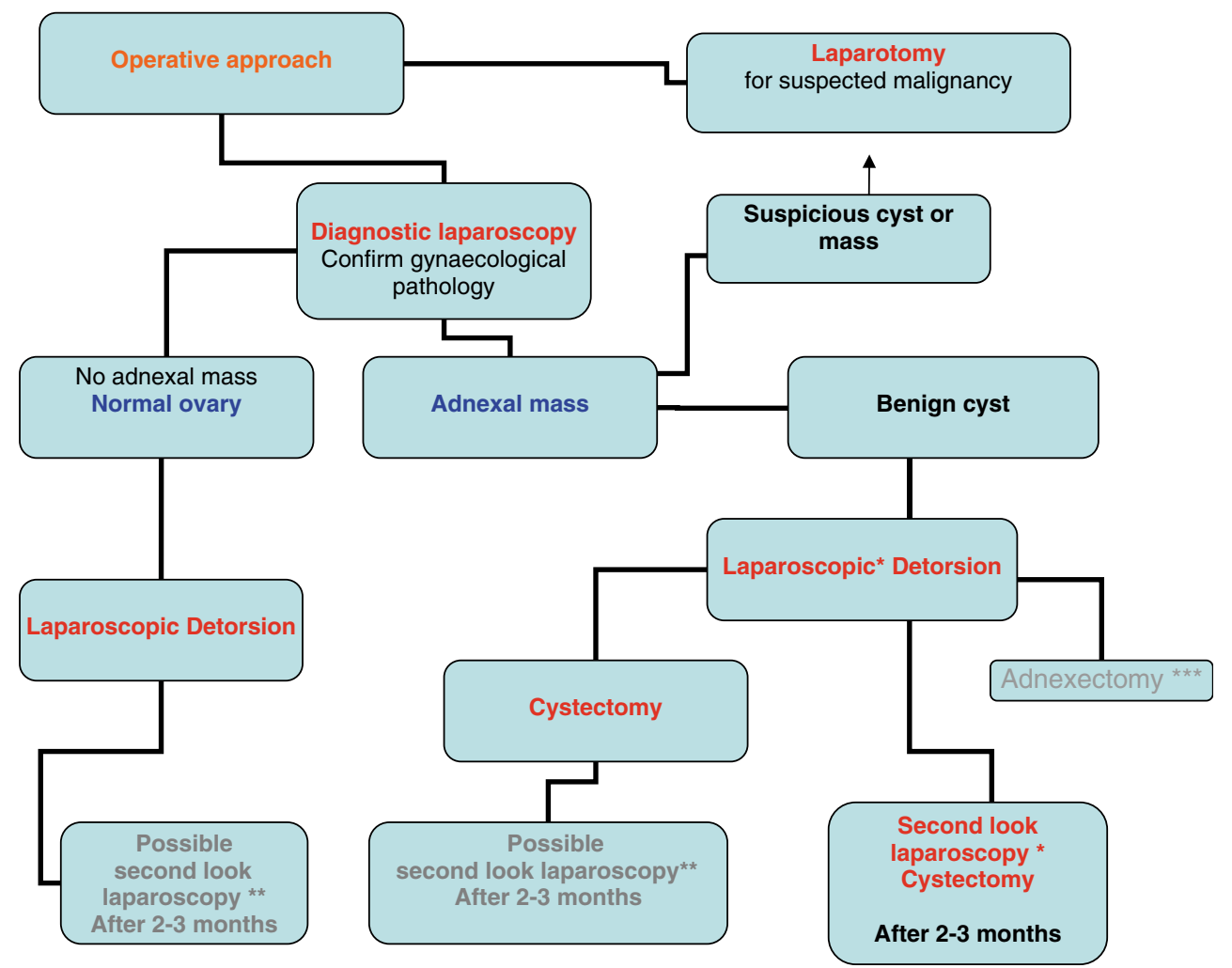

* Ovariopexy : uni or bilateral to consider in the first laparoscopy or in a second look check up

** Ovarian biopsy, possible ovarian cryopreservation in the future (?)

*** In case of non recovering gangrenous ovary or complication during operation 
the context of an absence of adnexal mass, observation may be indicated without the necessity of an operative approach. If there is any suspicion of malignancy associated with an ovarian mass, then laparotomy should be organised in coordination with a multidisciplinary team that includes a paediatric oncologist surgeon or gynaecologist [17]. In all the other cases, diagnostic laparoscopy is the method used to confirm directly the status of the adnexa and provide the diagnosis. Classification of three stages can be applied, depending on the laparoscopic aspect of the adnexa [18]: Stage A corresponds to an adnexal torsion with mild lesions and immediate recovery after detorsion. Stage B corresponds to severe ischemia with dark red or black colour of the adnexa, recovering partially after detorsion. Stage C corresponds to gangrenous ovary with no recovery after detorsion.

Depending on the laparoscopic findings, management should include the following steps (see Fig. 1), keeping in mind that "untwisting" should be attempted initially.

- If the ovaries are normal without cystic mass, detorsion is what needs to be considered next. The tube and adnexa can be very fragile because of the associated oedema, especially for stage C [18]. Detorsion, however, needs to be done with a gentle technique to protect the tissue. Trauma or injury to this tissue can occur at any time. Experienced surgeons and adequate assistance is mandatory (four-port access). The degree of difficulty is greater when the adnexa have multiple spires with more oedematous tissue. Sometimes, complete amputation is necessary at this stage (necrotic or gangrenous tissue) where the twisted adnexa is separated from the principal body of the tube [18]. During detorsion, satisfactory haemostasis is essential, and vigilance is necessary in all cases in order to avoid trauma. Tissue is smaller in young children and appropriately adapted instruments must be used. Initial subjective appearance of the ovary and tube does not correlate with the degree of ischaemia and potential ovarian-tissue damage. Thirtysix to 72 hours of ischemia is more highly correlated with a gangrenous appearance of the adnexa [7, 18], as seen from retrospective time evaluation from the initial signs of torsion until operation and additional information from animal models [19]. Great effort should be taken to apply conservative surgical management. Directly proceeding to oophorectomy or adnexectomy should be avoided, because after detorsion, the untwisted adnexa may progressively change colour due to the improvement in vascularity. The colour may change from ischaemic to a more normal pink shade. This is an empirically good sign of revascularisation. It is preferable to wait for up to $10 \mathrm{~min}$ or, in some cases, $15 \mathrm{~min}$ and then review the colour and appearance of the ovary and tube [18]. The tube will usually recover faster than the ovary, which may recover later even if its aspect is still dark grey at the end of the procedure. If there is no recuperation in colour with any other change, it is unlikely that revascularisation is achievable, and some surgeons perform adnexectomy only at this point (necrotic colour of the tube). For mild to severe ischaemic lesions, the conservative approach of detorsion is safe and has shown promising results for recovery of ovarian function [18]. If safe surgery is not possible or tissues are very friable, salpingectomy or oophorectomy (rarely) may be indicated.

- If there is an ovarian cyst causing the torsion, cystectomy with detorsion should be performed in one procedure, taking into consideration the volume and nature of the ovarian mass and whether or not haemorrhage and oedema are present. Surgeons may postpone the cystectomy until a second-look laparoscopic evaluation after 2 months. Attempting a later cystectomy theoretically preserves more ovarian tissue (less oedematous), with a smaller risk of trauma during the operation [20]. However, leaving a large cyst without initial cystectomy has the potential risk of torsion recurrence. On the other hand, a cystectomy with detorsion may be complicated by severe bleeding and the risk of proceeding to adnexectomy. In this case, a reasonable approach and available expertise play significant roles in the decisionmaking process.

- A second look laparoscopy 2-3 months postdetorsion could evaluate ovarian recovery [18]. The ovarian aspect (colour and volume) can be seen directly and compared with what was noted previously. The affected ovary tends to be smaller in volume (regression of oedema), and the colour usually remains whitish. Ovarian biopsy could be done at this point in order to confirm healthy cortex with follicles. Second-look laparoscopy should be used for research purposes only.

\section{Ovariopexy}

Ovariopexy [18] is indicated in cases of solitary adnexa torsion, torsion recurrence, abnormal ovarian ligaments, bilateral torsion, unilaterally affected tube and ovary with asynchronous torsion. The principal of ovariopexy techniques is to stabilise the adnexa in order to prevent torsion recurrence and possibly to correct anatomical hypermobility of the tube and ovary [21]. Patients with normal ovaries generally have abnormal ovarian ligaments, and ovariopexy is necessary.

To determine the need of ovariopexy in a patient with a twisted cystic ovary, the status of the contralateral adnexa should be determined. If the utero-ovarian ligament of the 
untwisted ovary is as long as the one of the twisted ovary, then the ligaments are abnormal and a bilateral ovariopexy is necessary. If the utero-ovarian ligament of the untwisted ovary is much shorter than that of the twisted ovary, then unilateral ovariopexy may be performed $[22,23]$. However, bilateral ovariopexy often is performed in such young patients in order to avoid any possible recurrence.

There is debate regarding whether preventive use of ovariopexy should be taken in all cases or only in cases of torsion recurrence [10]. It is unknown what effect (if any) these techniques may have to future fertility and whether they provoke a significant anatomical disturbance or associated chronic pain [9]. Ovariopexy is a questionable option in cases of normal adnexa without apparent anatomic reasons for torsion.

\section{Ovarian biopsy and cryopreservation of ovarian tissue}

Ovarian cryopreservation may be used more frequently in the future for possible ovarian-tissue transplantation. For the moment, these techniques are used for patients who will have chemotherapy that will affect both ovaries. Cryopreserved ovarian tissue can be used in women cured from cancer in order to reestablish ovarian function and possible fertility. Theoretically, ovarian biopsy and cryopreservation during a second-look laparoscopy could be done in cases of torsion in which there is only one remaining ovary with ovariopexy. Such application has not as yet been reported.

\section{Laparotomy versus laparoscopy}

Laparotomy was the usual surgical approach in all cases. It has now been more than 10 years since laparoscopy was used for the management of adnexal torsion [9, 24]. The laparoscopic approach combines numerous benefits in comparison with laparotomy, such as less postoperative pain and possible psychological stress for the young girl, quicker recovery-within $48 \mathrm{~h}$ - and return to home, faster assumption of physical activities and schooling and less stress for the families and easier parental support to their child.

Photos and video recordings during laparoscopic procedures become more and more a part of the daily practice in gynaecology [25]. Photographic illustrations have great value for future studies and research in cases of primary and secondary adnexal laparoscopic evaluation. Photos help the counselling of parents and the child: the surgeon can more easily demonstrate and explain the operating technique and justify indication for surgery.

There is little analogy between the results of laparoscopic surgery between adults and children. No postoperative complications from thrombotic embolus after detorsion were reported in recent literature [18]. Transitional subfebrile status is noted occasionally.

\section{What we do not yet know}

The criteria for successful treatment are variable in the management of adnexal torsion. The main objective is to deal with pain and prevent recurrence of torsion while preserving ovarian function for the adult years. Postoperative parameters that have been used to assess ovarian recovery include colour Doppler studies, US studies with detection of follicular activity in the affected ovary and observed pregnancy rates in adult life. However, all these are indirect ways of evaluating the function of the affected ovary and its recovery. Direct evaluation could be done by biopsy in a second-look laparoscopy in order to detect presence or absence of follicles. Operating on young girls a second time for research purposes is not always ethical, however.

It is very difficult to evaluate possible postoperative complications such as subfertility and pelvic adhesion formation after cystectomy or ovariopexy. Little is known about pain occurrence in subsequent years and whether or not it is related to the intervention. Adnexal torsion is a very rare event, especially in premenarcheal girls. Most published results give information from heterogeneous cases (different ages that range from very young girls, premenarcheal girls, teenagers and even childbearing women). These heterogeneous age-related cases and variation of pathologies do not allow accurate conclusions as to what the best management may be. Future data and a systematic approach towards these rare cases will help gynaecological endoscopists optimise their theurapeutical options.

The use of CT scan or MRI depends of the availability, cost and access in each hospital. It is quite difficult to evaluate their use in a comparative way. It is unclear whether $\mathrm{CT}$ or MRI findings can determine haemorrhagic infarction and whether there is a chance of ovarian preservation. The pathology may vary with or without adnexal mass [16]. More data may be necessary in the future in order to distinguish between cost efficacy of CT scans versus MRI and prognosis. Still, in most hospitals, CT scan is realised faster than MRI.

The fact that the right adnexa is more often reported as affected can be related to anatomical reasons (the left colon may protect against left torsion) or because there is a "faster" surgical intervention if the pain simulates right appendicitis. We do not know whether there is more significant damage to the left adnexal torsion due to possible delay of appropriate diagnosis.

Management depends on who will see the young girl initially. It seems that a more conservative laparoscopic 
approach is followed by gynaecologists, with less laparotomies being performed than by general surgeons $[3,7]$.

\section{Conclusion}

Recent advances in laparoscopic techniques, imaging and experience place conservative laparoscopy as the first theurapeutic approach in case of adnexal torsion in premenarcheal girls. In the future, cohorts of observational studies are necessary to determine long-term complications after detorsion. The algorithm that we propose may help the counselling of these patients and prioritise the different surgical options for each case.

\section{References}

1. Holland AJA, Barker AP, Robins PD (1995) Isolated torsion of the fallopian tube in a premenarcheal girl. Pediatric Surg Int 11:47-49

2. Krissi H, Salev J, Bar-Hava I, Langer R, Heran A, Kaplan B (2001) Fallopian tube torsion: laparoscopic evaluation and treatment of a rare gynaecological entity. J Am Board Fam Pract 14(4):274-277

3. Bristow RE, Nugent AC, Zahurak ML, Khouzhami V, Fox HE (2006) Impact of surgeon specialty on ovarian conserving surgery in young females with an adnexal mass. J Adolesc Health 39:411-416

4. Berger J, Sauvage P (1970) Torsion of the ovary and adnexal in childhood. Ann Chir Inf 11:235-242

5. Spingland N, Ducharme JC, Yazbeck S (1989) Adnexal torsion in children. J Pediatr Surg 24:974-997

6. Davies AJ, Feins NR (1990) Subsequent asynchronous torsion of normal adnexal in children. J Pediatr Surg 25:687-689

7. Emonts M, Doornewaard H, Admiraal, CJ (2004) Adnexal torsion in very young girls: diagnostic pitfalls. Eur J Obstet Gynecol Reprod Biol 116:207-210

8. Holland AJ, Barker AP, Robins PD (1995) Isolated torsion of the fallopian tube in a premenarcheal girl. Pediatr Surg Int 11:47-49

9. Steyaert H, Meynol F, Valla JS (1998) Torsion of the adnexa in children: the value of laparoscopy. Pediatr Surg Int 13:384-387

10. Crouch NS, Cunter AS, Creighton SM (2003) Ovarian torsion: to pex or not to pex? Case report and review of literature. J Pediatr Adolesc Gynecol 16:381-384
11. Breech LL, Hillard PJA (2005) Adnexal torsion in pediatric and adolescent girls. Curr Opin Obstet Gynecol 17:483-489

12. Taskin O, Birincioglu M, Aydin A et al (1998) The effects of twisted ischaemic adnexa managed by detorsion on ovarian viability and histology: an ischaemia-reperfusion rodent model. Hum Reprod 13:2823-2827

13. Van Kerkhove F, Cannie M, Op de beedk K, Timmerman D, Pienaar A, Smet MH, Bielen D, Vanbeckevoort D, Dymarkowski S (2006) Ovarian torsion in a premenarcheal girl: MRI findings. Abdom Imaging [Epub ahead of print]

14. Bader T, Ranner G, Haberlik A (1996) Torsion of a normal adnexa in a premenarcheal girl: MRI findings. Eur Radiol 9 (6):704-706

15. Ben-Ami M, Perlitz Y, Hadded S (2002) The effectiveness of spectral and colour Doppler in predicting ovarian torsion: a prospective study. Eur J Ostetr Gynecol Reprod Biol 104:64-66

16. Rha SE, Byun JY, Jung SE, Jung JI, Choi BG, Kim BS, Kim H, Lee JM (2002) CT and MR imaging features of adnexal torsion. Radiographics 22:283-294

17. Woo YL, MacDougall J, Latimer J, Cawford R (2005) Laparoscopic management of adnexal masses in adolescent females: a multidisciplinary approach. Gynecol Surg 2:227-230

18. Canis M, Mage G, Wattiez A, Manhes H, Pouly JL, Bruhat MA (2003) Laparoscopic management of adnexal torsion. Chapter 20 pp 209-212 Atlas of operative laparoscopy and hysterectomy. Second edition. Parthenon publishing group

19. Kaleli B, Aktan E, Gezer S, Kirkali G (2003) Reperfusion injury after detorsion of unilateral ovarian torsion in rabbits. Eur J Obstet Gynecol Reprod Biol 110:99-101

20. Cohen SB, Wattiez A, Seideman DS, Goldenberg M, Admon D, Mashich S, Oelsner G (2003) Laparoscopy versus laparotomy for detorsion and sparing of twisted ischemic adnexa. J Soc Laparoendosc Surg 7:295-299

21. Jardon K, Bothschorisvili R, Rabischong B, Rivoire C, Nohuz E, Houlle C, Pouly JL, Mage G, Canis M (2006) How I perform an ovariopexy after adnexal torsion. Gynecol Obstet Fertil 34:529-530

22. Mage G, Canis M, Manhes H, Pouly JL, Bruhat MA (1989) Laparoscopic management of adnexal torsion. A review of 35 cases. J Reprod Med 34(8):520-524

23. Manhes H, Canis M, Mage G, Pouly JL, Bruhat MA (1984) Laparoscopy in the diagnosis and treatment of adnexal torsion. J Gynecol Obstet Biol Reprod (Paris) 13(7):825-829

24. Cohen Z, Shinhar D, Kopernik G, Mares AJ (1996) The laparoscopic approach to uterine adnexal torsion in childhood. J Pediatr Surg 31(11):1557-1559

25. Panayotidis $C$ (2005) Illustrations in gynaecology: still controversial? Gynecological Surgery 2(3):165-168 